REVUE BIMESTRIELLE

CENTRE D'INFORMATION

ET D'ÉTUDES

SUR LES MIGRATIONS

INTERNATIONALES
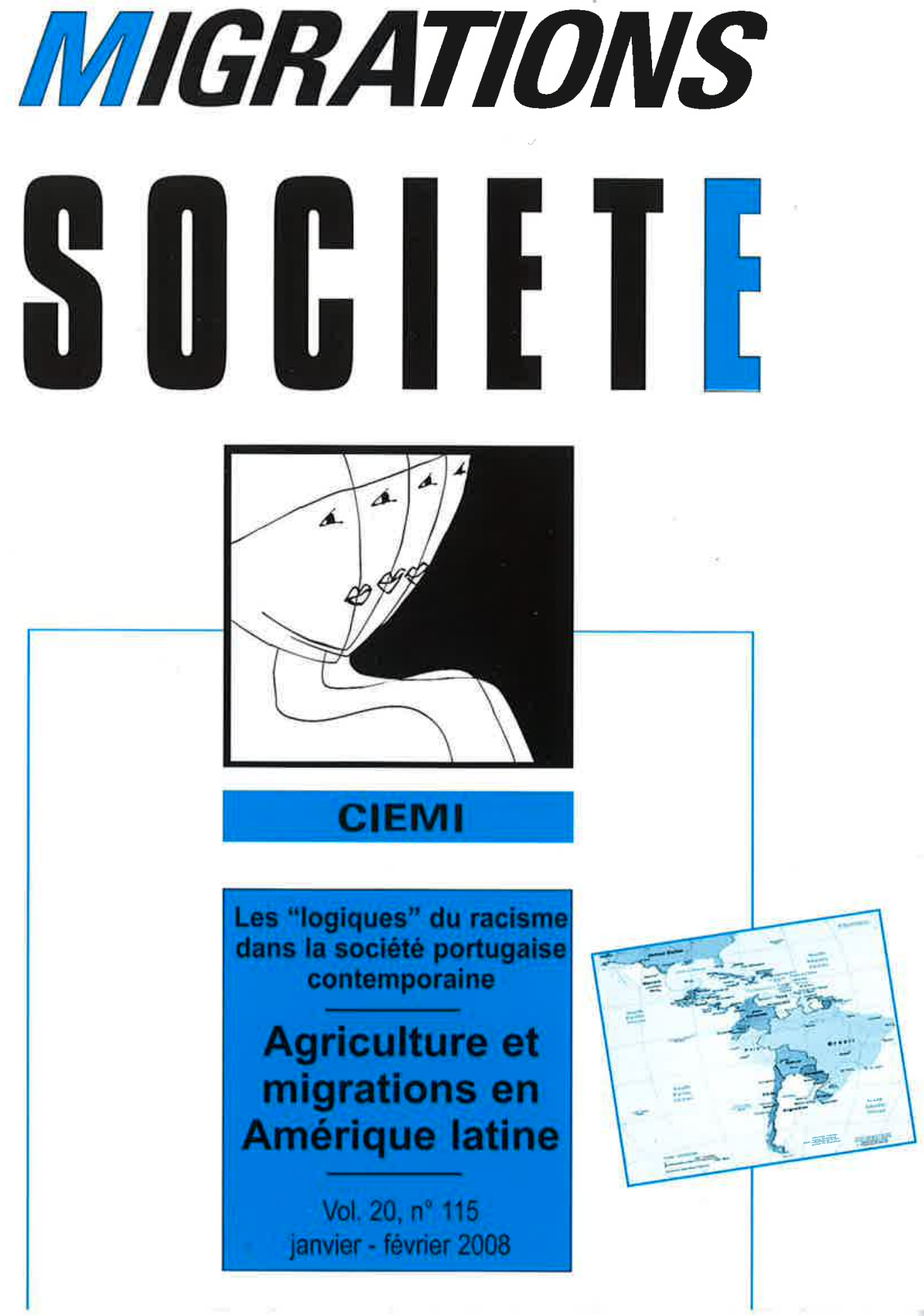


\title{
LES “LOGIQUES" DU RACISME DANS LA SOCIÉTÉ PORTUGAISE CONTEMPORAINE
}

Joc̃o Filipe MARQUES *

\begin{abstract}
«Il ne faut donc pas confondre la volonté de dominer avec le désir phobique de pur rejet: la première tend à s'accomplir dans l'exploitation économique, le second dans l'expulsion ou l'extermination ».
\end{abstract}

Pierre-André Taguieff ${ }^{1}$

Le Portugal ne semble pas être une exception en matière d'attitudes et de comportements racistes, observables dans la plupart des pays d'Europe. Toutefois, les événements à caractère raciste que rapportent les médias depuis une quinzaine d'années mettent en cause un des traits les plus caractéristiques de la mythologie nationale portugaise : le "non-racisme" et la "tolérance" des Portugais à l'égard des autres peuples. Par ailleurs, ces événements semblent n'être que la partie émergée de l'iceberg, laissant deviner des phénomènes beaucoup plus répandus : les conduites discriminatoires inscrites dans le fonctionnement de la société, l'hostilité quotidienne, les préjugés, la violence du langage, la ségrégation spatiale, etc.

Sans céder aux tentations du soupçon généralisé ni à celles de la dénonciation gratuite, un ensemble de questions mérite d'être posé à ce propos : comment se manifeste le racisme dans la société portugaise contemporaine et à quelles logiques obéit-il ? Quelles sont ses sources actuelles et historiques?

* Sociologue, Faculdade de economia, Universidade do Algarve, Portugal. Contact : jfmarq@ualg.pt. Le présent article reprend quelques idées développées dans la thèse de doctorat en sociologie de l'auteur intitulée Je ne suis pas raciste mais... Du "non-racisme" portugais aux deux racismes des Portugais, Paris : EHESS, 2004, 380 p. La recherche a bénéficié de l'appui d'une bourse de la Fundação para a Ciência e Tecnologia et du Fonds social européen dans le cadre du $1 /{ }^{\circ}$ programme-cadre européen de soutien.

1. TAGUIEFF, Pierre-André, Le racisme, Paris : Éd. Flammarion, 1997, 127 p. (cf. pp. 60-61).

Migrations Société 
Le présent article tente de fournir quelques pistes permettant une meilleure intelligibilité des phénomènes racistes actuellement observables dans la société portugaise, une intelligibilité permettant de mieux agir ou réagir politiquement contre ce type de phénomènes. Pour cela, nous ferons appel à deux approches complémentaires. D'une part, nous analyserons les contenus d'un ensemble d'entretiens réalisés avec des responsables d'associations d'immigrés ou de Tsiganes ainsi que d'organisations antiracistes, des responsables politiques et institutionnels, des syndicalistes et des citoyens portugais et étrangers. Cette démarche s'est avérée appropriée pour saisir les modèles et les logiques du racisme dans la société portugaise contemporaine, tout en donnant la parole à leurs victimes et à ceux qui leur sont proches. D'autre part, nous exploiterons plusieurs autres sources d'information et nous analyserons, à la lumière de l'approche sociologique du racisme, un corpus de recherches fondées sur des paradigmes théoriques et des approches empiriques diverses consacrées aux questions relatives à l'immigration, à l'ethnicité, à l'identité des immigrés et à l'"insertion" des Tsiganes, aux modes de vie des minorités ethniques.

\section{Les "logiques" du racisme}

La littérature est presque unanime à affirmer que le racisme obéit à deux logiques étroitement liées mais passibles d'un découpage idéaltypique : la logique inégalitaire et la logique différentialiste ${ }^{2}$.

La logique inégalitaire de l'infériorisation inscrit les groupes dominés dans la trame des relations sociales à condition qu'ils occupent les places qui leur sont destinées - toujours les plus dévalorisées - et qu'ils se cantonnent dans les espaces socialement méprisés. Cette forme de racisme, nettement liée aux systèmes de stratification, fait plus fréquemment appel aux arguments des théories biologiques, soulignant l'inégalité des attributs naturels entre "racisants" et "racisés" de façon à rationaliser et à légitimer soit des comportements discriminatoires, soit le maintien des systèmes sociaux fondés sur la domination et l'exploitation. Les paradigmes historiques des systèmes sociaux fondés

2. Voir, entre autres, TAGUIEFF, Pierre-André, La force du préjugé. Essai sur le racisme et ses doubles, Paris : Éd. La Découverte, 1987, 645 p. ; BAL.IBAR, Étienne; WALLERSTEIN, Immanuel, Race, nation, classe. Les identités ambiguês, Paris : Éd. La Découverte, 1988, 310 p. ; WIEVIORKA, Michel, L'espace du racisme, Paris : Ed. du Seuil, 1991, 255 p. ; SCHNAPPER, Dominique, La relation à I'Autre au coeur de la pensée sociologique, Paris : Éd. Gallimard, 1998, 557 p. Cette distinction semble avoir été formulée pour la première fois par HERSCH, Jeanne, dans l'article "Sur la notion de race", Diogéne, $n^{\circ} 59$, mars 1967, pp. 125-142. 
sur le racisme inégalitaire ou d'infériorisation se trouvent, par exemple, dans la société du sud des États-Unis jusqu'à la guerre de Sécession ou dans les sociétés coloniales. Suivant la construction idéal-typique proposée par Pierre-André Taguieff, on peut expliciter cette logique selon la série "hétéroracisation/inégalité/domination/exploitation " ${ }^{3}$.

Selon la deuxième logique du racisme, la logique différentialiste, le groupe victime est perçu comme une menace endogène qu'il faut écarter et on ne lui reconnaît aucune place dans le système social. Tout au contraire, cette logique engendre le rejet, le désir d'exclusion, de mise à distance et, à la limite, d'expulsion ou de destruction. Tout en naturalisant les attributs du groupe victime, la logique différentialiste souligne ses traits culturels ef identitaires, qui sont cependant perçus comme des entités rigides, substantielles, essentielles, donc comme des traits permanents. L"Autre" est considéré comme "polluant", comme un "corps étranger" qui porte atteinte à l'homogénéité culturelle et aux valeurs identitaires du "Nous". Il s'agit d'une forme de racisme qui non seulement met en évidence l'incommensurabilité des différences culturelles entre les groupes concernés, mais qui souligne également leur irréductibilité ef donc leur incompatibilité.

Les paradigmes historiques de cette logique de "racisation" sont, naturellement, l'antisémitisme nazi et, selon certains auteurs, le néoracisme européen à l'égard des populations issues de l'immigration. Selon la proposition de P.-A. Taguieff, le racisme différentialiste (I s'explicite par la série : autoracisation/différence/purification-épuration/extermination $1{ }^{4}$.

En ce qui concerne les objectivations sociales des deux logiques du racisme, on peut dire, de façon générale, que tandis que le racisme inégalitaire discrimine et exploiłe, le racisme différentialiste se traduit par la ségrégation ou l'expulsion. Or cette typologie des expressions du racisme est liée aux logiques mêmes du fonctionnement de la société. Selon Michel Wieviorka, " la distinction entre racisme de différence et racisme d'inégalité renvoie ò des figures, elles aussi beaucoup plus générales, que connaissent bien les sociologues, à deux grandes familles de problèmes qu'ils ont appris depuis longtemps à ne pas confondre. L'idée d'inégalité, en effet, n'est qu'une formulation, parmi d'autres, de la division de la société et de ses rapports de domination; celle de différence, au contraire, a trait à l'unité du corps social, ou d'un de ses sous-ensembles,

3. TAGUIEFF, Pierre-André, La force du préjugé. Essai sur le racisme et ses doubles, Paris : Éd. La Découverte, 1987, 645 p. (cf. p. 163).

4. Ibidem. 
que celle-ci soit désignée en termes larges de culture, de communauté et d'identité, ou en termes plus précis de nation, de religion et, à la limite, de race $11^{5}$.

\section{Le racisme dans la sociéłé porługaise}

\section{- Y a-t-il du racisme dans une société qui se pense comme} "non raciste"?

"Y a-t-il du racisme dans la société portugáise? ". Tous les interviewés ont été unanimes à répondre affirmativement à cette question, mais - paradoxalement et contrairement à ce qui pourrait être attendu - de façon générale, la plupart d'entre eux n'ont profité de l'entretien ni pour s'en indigner ni pour dénoncer sérieusement le fonctionnement de la société portugaise. Presque tous - Portugais, Tsiganes portugais, immigrés ou Luso-Africains - sont très conscients du fait que les Portugais pensent qu'il n'y a pas de racisme au Portugal ou qu'il y en a mais moins qu'ailleurs, moins que jadis, ou encore que les Portugais pratiquent une forme spéciale ef plus souple de racisme. Le racisme de la société portugaise est très souvent relativisé, mitigé ou comparé — de façon positive - avec celui pratiqué dans d'autres pays :

"Ie pense qu'il $y$ a du racisme au Portugal. Peut-être pas autant que dans d'autres pays, mais il y en a " [Olga Mariano, Tsigane, Association des femmes tsiganes portugaises, Lisbonne].

"Toutes les sociétés ont quelque chose de raciste. II n'y a pas de société à l'abri de ce phénomène. La société portugaise, quoiqu'elle ne soit pas comme d'autres sociétés, dont il est commun de dire qu'elles sont plus racistes et où il existe une discrimination plus déclarée, il est évident que dans la société portugaise il y a aussi du racisme " [António Veiga, Portugais, Association guinéenne de solidarité sociale, Lisbonne].

"S'il n'y avait pas de racisme dans la société portugaise cette association n'aurait pas de raison d'exister. II n'est peut-être pas si fort qu'il l'était quelques siècles auparovant ") [Bruno Gonçalves, Tsigane, Association tsigane de Coimbra, Coimbra].

"Bien sur qu'il y a du racisme [...]. Aujourd"hui, au Portugal, une discussion quelconque entre un Noir et un Blanc finit toujours dans le racisme. Pourquoi? Parce que faire des représailles sur un Noir s'est pratiquement institutionnalisé ")

5. WIEVIORKA, Michel, L'espace du racisme, op. cit., p. 92.

Vol. $20, n^{\circ} 115$

janvier - février 2008 
[Emílio Brazão, Cap-Verdien, Association des amis de la Rive Sud du Tage, Barreiro].

L'idée selon laquelle les Portugais pratiqueraient un racisme "spécial" et plus souple est très présente dans la plupart des esprits, mais il y a aussi pas mal d'individus qui se révoltent contre ce mythe et qui n'hésitent pas à le dénoncer.

"Le racisme, au Portugal, existe comme il existe n'importe où dans le monde. Je pense que les Portugais ont un grand souci d'affirmer sur-le-champ : "Je ne suis pas raciste" ou "Nous ne sommes pas des racistes". Je dois dire qu'il existe du racisme dans la société porfugaise comme n'importe où. Je me souviens d'une émission de télévision, un entretien dans RTP [Radio télévision portugaise-África, où la journaliste affirmait: "Nous, les Portugais, nous ne sommes pas racistes, nous n'avons pas une culture raciste". Moi, ce dont i'ai envie, c'est de dire à ces gens-là, de leur demander s'ils veulent changer de peau avec moi au moins pendant 24 ou 48 heures. Ça leur ferait voir s'il y a du racisme ou pas. Quand je dis que je changerais ma peau pour 24 heures, c'est parce que, alors, les gens sentiraient sans doute le racisme au jour le jour "I [António Tavares, Angolais, Association de défense des Angolais, Lisbonne].

"Les Portugais pensent qu'ils ne sont pas racistes. Ma théorie pour expliquer ça c'est qu'ils regardent l'histoire, et quand ils sont allés découvrir des choses qui éłaient déjà là, quand ils sont allés les conquérir, ils n'ont pas été aussi maurais que les Espagnols, les Français ou les Anglais; par conséquent ils ne sont pas aussi racistes que les Espagnols, les Français ou les Anglais )" [Mónica Frechaut, Luso-Africaine, Centre culturel africain, Setübal].

Comme on pourrait s'y attendre, ces témoignages coïncident avec les résultats d'autres recherches sociologiques et d'autres recueils d'entretiens sur le racisme au Portugal, comme les entretiens réalisés par Elsa Sertório ou Teresa Castro d'Aire. Là encore, quoique dénoncée, l'expérience du racisme est mitigée et l'histoire coloniale du pays est utilisée dans la justification de cette situation :

"Je pense qu'il y a du racisme au Portugal, mais je pense que le racisme au Portugal... On dit que tous les Portugais ont des mceurs douces et i'aime croire ò ça; je pense même que, en ce qui concerne le racisme, ils sont relativement doux. Je pense que les Portugais ont un type de racisme très spécifique. Ils sont relativement permissifs vis-à-vis de l'entrée de personnes d'autres ethnies dans la société — relativement — mais en ce qui concerne les valeurs... ") [Anlid Costa, Luso-Africain] $]^{6}$.

6. Cité par SERTÓRIO, Elsa, O livro negro do racismo em Portugal, Lisboa : Ed. Dinossauro, 2001, 192 p. (cf. p. 38). 
" Oui, il y a du racisme. Contre les Tsiganes et contre d'autres "races". On parle beaucoup des Découvertes et du mélange des "races", mais il existe toujours beaucoup de racisme au Portugal, même s'il s'agit d'un racisme caché ") [João Laços, Tsigane] ${ }^{7}$.

Quelques enquêtes sociologiques menées à partir de questionnaires confirment tout cela et proposent une certaine quantification des résultats. Selon les données de la recherche sur les " perceptions de racisme au quotidien " réalisée par Fernando Luis Machado auprès des immigrés d'origine guinéenne, $63 \%$ de ceux qui ont répondu au questionnaire affirment qu'il y a beaucoup de racisme au Portugal, 32,7\% qu'il y en a, " mais peu "), et seulement 1,7\% répondent qu'il n'y a pas de racisme au Portugal ${ }^{8}$. Les données de la recherche d'Ana SaintMaurice sur les représentations sociales des immigrés cap-verdiens à l'égard des Portugais corroborent cette même image. Selon cette enquête, $66,5 \%$ des personnes ayant répondu au questionnaire trouvent que la " majorité des Portugais est raciste $\|^{9}$.

Le racisme, quoi qu'en pensent les Portugais, fait partie des nouveaux enjeux auxquels est confrontée la société portugaise. II faut dès lors distinguer quelles sont les catégories sociales qui constituent ses principales victimes, saisir les logiques auxquelles il obéit et essayer de discerner ses origines sociétales et historiques.

\section{- Qui sont les principales victimes ?}

Encore une fois, on constate une relative unanimité chez les interviewés lorsqu'il s'agit de savoir qui sont les premières victimes du racisme, à savoir les Tsiganes et les personnes d'origine africaine :

"Ce sont les Tsiganes, les Nègres, les victimes du racisme des Portugais" [Luís Miguel Grade, commerçant, Tsigane, Lisbonne].

"Les immigrés et les Tsiganes sont les plus maltraités " [Verónica Mendes, Luso-Africaine, étudiante, Lisbonne].

7. Cité par AlRE, Teresa Castro d', O racismo, Lisboa : Ed. Temas da Actualidade, 1996, 148 p. (cf. pp. 3637).

8. Cf. MACHADO, Fernando Luís, "Contextos e percepçōes de racismo no quotidiano", Sociologia Problemas e práticas, $n^{\circ} 36$, setembro 2001, pp. 53-80 (voir p. 56).

9. SAINT-MAURICE, Ana, Identidades reconstruidas. Cabo-verdianos em Portugal, Oeiras : Ed. Celta 1997,171 p. (cf. p. 143).

Vol. $20, n^{\circ} 115$

janvier-févier 2008 
" La communauté tsigane continue d'être la plus discriminée dans notre pays. C'est une réalité ") [Bruno Gonçalves, Tsigane, Association tsigane de Coimbra, Coimbra].

En tout cas, le racisme vécu par les Tsiganes est beaucoup plus souligné que celui qui touche les immigrés. Même les interlocuteurs d'origine africaine perçoivent les Tsiganes comme les victimes du racisme le plus intense :

" D'abord, ce sont les Tsiganes, après ce sont les immigrés africains récemment arrivés au Portugal - ceux qui ne savent pas encore comment ça marche, qui ne savent pas comment sont les gens et qui ne maîtrisent pas la langue - et après ce sont les jeunes; ceux qui sont nés ici mais qui n'appartiennent ni à "ici" ni à "là-bas". Mais en premier lieu, ce sont les Tsiganes, ils sont les plus discriminés )" [Mónica Frecheaut, Luso-Africaine, Centre culturel africain, Setúbal].

Les acteurs institutionnels, notamment les dirigeants des associations de lutte contre le racisme et le Haut Commissaire à l'immigration et aux minorités ethniques, confirment cette perception des acteurs sociaux :

"I Les victimes [du racisme] sont d'abord les pauvres; les Tsiganes parce qu'ils sont paurres, les Africains non seulement parce qu'ils sont noirs mais parce qu'ils sont pauvres aussi $/$ [Elsa Sertório, Association solidariedade imigrante, Lisbonne].

" Je dirais que [les victimes du racisme] ce sont d'abord les Tsiganes et après les immigrés africains ") [José Leitão, Portugais, Haut Commissaire à l'immigration et aux minorités ethniques].

Plusieurs autres sources donnent des résultats semblables. Selon l'enquête menée par Joana Miranda sur les stéréotypes véhiculés par les Portugais à l'égard des groupes ethniques ${ }^{10}$, les ressortissants des PALOP ${ }^{11}$ et les Tsiganes sont les victimes des stéréotypes les plus négatifs. Par ailleurs, l'enquête menée par Maria loannis Baganha aboutit à la conclusion suivante : quelque $70 \%$ des Portugais n'aimeraient pas habiter près d'un campement de Tsiganes et $40 \%$ d'entre eux n'aimeraient pas vivre dans un quartier de "Noirs"12.

10. Cf. MIRANDA, Joana, Grupos étnicos em Portugal. Os estereótipos dos "portugueses", mémoire de maîtrise en relations interculturelles, Lisboa : Universidade Aberta, 1994, 192 p. (photocopié).

11. Pays africains de langue officielle portugaise.

12. Cf. BAGANHA, Maria loannis, Immigrant insertion in the informal economy: the Portuguese case, Coimbra : CES, Universidade de Coimbra, 1996, 126 p. (voir pp. 104 et 140).

Migrations Société 
- Comment se manifeste le racisme dans la société portugaise ?

Tel qu'il est vécu et qu'il est décrit par ses victimes et par ceux qui leur sont proches, le racisme se manifeste par deux dimensions qui varient selon le groupe qui en est victime. D'une part, les Tsiganes se perçoivent comme les cibles d'un racisme aux contours différentialistes, essentiellement rural, qui se concrétise par un violent rejet de la part des villageois. Dans ces milieux ruraux, la ségrégation, le désir d'éloignement et les conduites d'écartement sont prépondérants. D'autre part, les populations issues de l'immigration africaine sont plutôt la cible d'un racisme de type inégalitaire nettement urbain dont les principales manifestations sont l'infériorisation ef la discrimination, éventuellement la violence verbale ou le harcèlement.

\section{- Le racisme inégalitaire à l'égard des immigrés}

Les manifestations de racisme à l'égard des immigrés d'origine africaine semblent imprégner insidieusement tous les domaines de la vie sociale. II ne s'agit pas, sauf quelques exceptions, d'agressions physiques, de manifestations spectaculaires de violence raciste ou de persécutions brutales. Les dénonciations des interviewés portent surtout sur les discriminations vécues dans les domaines de l'emploi et de la recherche de logement, dans les transports en commun, dans les lieux de consommation ou de loisirs. La couleur de la peau continue d'être perçue comme un signe d'infériorité qui "justifie" le traitement différentiel des populations issues de l'immigration africaine :

"La couleur de la peau joue un rôle très important dans la façon dont les gens sont traités. On voit ça essentiellement au niveau du travail et du logement. Je n'en dirais pas autant au niveau du système éducatif parce que là, en principe, il n'y aurait pas de grands problèmes. On trouve des cas de personnes qui sont en grande difficulté pour lover une maison d̀ cause de la couleur de leur peau " [Pedro Tavares, Portugais, consultant juridique au Conseil portugais pour les réfugiés, Lisbonne].

"Le racisme dans la société portugaise fient surfout à la discrimination en termes d'égalité des chances, devant l'emploi ou dans l'accès aux biens et services. Bien plus que le racisme des offenses ou des agressions, il s'agit de cette discrimination... Dans des choses aussi simples que lover une chambre aux personnes d'origine africaine... si les gens ont une couleur de peau déterminée... c'est beaucoup plus difficile " [João Lázaro, Portugais, Association portugaise de soutien à la victime, Lisbonne]. 
Selon la plupart des interviewés, ce traitement différentiel est omniprésent et traverse presque toutes les dimensions des rapports interpersonnels :

"Le racisme, on l'aperçoit dans le supermarché, dans la rue, dans le centre commercial. On le voit; les attitudes racistes au quotidien sont flagrantes. Ici, dans le quartier, par exemple, si une voiture est volée, la réaction immédiate est : "C'est les Nègres". II y a quelques jours, on a cambriolé cinq ou six Fiał Uno et la réaction a été : "C'est les Nègres", et pourtant quelqu'un a dit: "Non, i'ai vu un homme blanc en costume avec un pied-de-biche dans les mains et i'ai appelé la police". La police n'est arrivée qu'à cinq heures du matin et n'a pas attrapé l'homme. II n'avait rien à voir avec le quartier, mais la première réaction a été : "C'est les Nègres"... ") [Olga, Portugaise, Association viver no mundo, Sacavém].

"C'est très difficile pour moi de parler du racisme parce qu'il est... systématique. C'est tous les jours. Tous les jours je dois justifier des petits comportements [des jeunes Luso-Africains] que les gens ne comprennent pas. J'en ai marre des scènes de racisme qui ont lieu [contre des jeunes LusoAfricains]. Tous les jours il y a quelque chose de bizarre... à la boulangerie, au café, quand on va à la plage... II y a tant d'exemples... dans le Centro de Emprego [Centre pour l'emploi]... Parfois les choses semblent aller très bien, et fout d'un coup il y a quelqu'un qui dit: "Ah !, les Nègres, c'est toujours les Nègres" [...]. À l'école ou où que ce soit, c'est toujours la même chose : "Tu es idiot, tu es bêfe". II n'y a jamais d'éloges [...]. C'est toujours les traits négatifs. II n'y a jamais des trucs positifs à retirer de leurs expériences, rien. Ils [les jeunes Luso-Africains] sont toujours bêtes, toujours idiots, toujours moches, toujours des cochons, ils sentent toujours mauvais... C'est ça tout le temps... " [Maria João Marques, Portugaise, Association Unis du Cap-Vert, Amadora].

L'infériorisation et le paternalisme typiques des rapports sociaux coloniaux demeurent présents dans la société portugaise :

"Il est évident que le racisme portugais a beaucoup à voir avec le paternalisme; le Portugal a été un pays colonisoteur et il est évident que dans les rapports avec les Africains il avait toujours une bonne partie de paternalisme. II n'est pas facile de regarder l'Autre en tant qu'égal. La société n'est pas encore préparée pour ça ò $100 \%$ " [António Veiga, Portugais, Association guinéenne de solidarité sociale, Lisbonne].

"Les emplois pour les Noirs au Portugal, ça se résume ò deux ou trois domaines: pour les hommes c'est le bátiment, pour les femmes le nettoyage ou les cuisines des restaurants. Parce qu'il s'agit de lieux qui ne sont pas visibles. Et si on se demandait où sont les Noirs qui ont étudié dans les universités? Parce qu'il y a beaucoup de Noirs qui éfudient, mais ils ne sont nulle part. IIs continuent à travailler dans les restaurants ou dans le bâtiment. Un des exemples de racisme au travail que je peux vous donner c'est une 
histoire qui s'est passée avec moi. J'avais 17 ou 18 ans et i'avais répondu à une annonce de travail pour transporter des meubles. Quand i'ai téléphoné on m'a dit oui, qu'ils avaient besoin de quelqu'un, mais quand je suis arrivé, la personne qui m'a reçu m'a dit qu'elle ne pourrait pas m'accepter parce que i'étais noir, qu'il s'agissait de transporter des meubles chez des clients et que, étant noir, je pourrais faire fuir la clientèle ") [António Tavares, Angolais, Association de défense des Angolais, Lisbonne].

Cette exigence de l'invisibilité des populations d'origine africaine n'est pas exclusive du domaine de l'emploi, elle est observable aussi dans les lieux de loisirs ou d'habitation. La plupart des Noirs sont censés ne pas habiter ni fréquenter les mêmes endroits que le reste de la population :

" Dans les lieux de loisirs... voilà un type de discrimination avec laquelle je ne perds pas mon temps... Moi-même j'ai déjà... enfin... Il y a un restaurant tout près d'ici où, les trois ou quatre fois où i'y suis allé avec ma femme, ils m'ont toujours dit que c'était plein. Même avec des tables vides. J'ai déjà essayé plusieurs fois. Chaque fois que je vais à ce restaurant-là ils me disent: "C'est plein, il n'y a pas de place" ") [Adriano Malalane, Mozambicain, Association mozambicaine, Lisbonne].

"Quand on entre dans certains endroits qui habituellement ne sont pas fréquentés par des Noirs, on sent le problème du racisme; ou alors on nous dit: "C'est des joueurs de foot", car il y a des endroits où les seuls clients noirs sont des joueurs de football, parce qu'ils ont de l'argent" "António Tavares, Angolais, Association de défense des Angolais, Lisbonne].

Pour ne pas être soumises à de telles vexations ces personnes finissent par éviter ce type de lieux, voire le contact avec les autochtones, ne fréquentant alors que les espaces qui leur sont réservés. La discrimination donne très souvent lieu à des phénomènes d'autoségrégation :

"Non, il n'y a pas de discrimination dans les discothèques, parce qu'ils [les jeunes Luso-Africains] ne fréquentent que des discothèques pour Noirs " [Maria João Marques, Portugaise, Association Unis du Cap-Vert, Amadora].

Au stigmate de la couleur de la peau s'ajoute celui du quartier d'habitation. La méfiance et le stéréotype de la marginalité sont si puissants que les jeunes Luso-Africains qui habitent dans des quartiers dégradés des banlieues de Lisbonne et de Porto doivent, par exemple, inventer des fausses adresses pour trouver un emploi :

" On donne une fausse adresse pour obtenir un emploi. À moi il m'est déjà arrivé de donner l'adresse de ma tante qui habite le centre-ville. Ils emploient des Noirs mais ne veulent pas les gens de la banlieue " [Verónica Mendes, Luso-Africaine, étudiante, Lisbonne]. 
Êre d'origine africaine et habiter dans certains quartiers marqués négativement est immédiatement perçu comme un signe de marginalité :

"I Je peux vous donner l'exemple flagrant d'un garçon qui habite près de chez moi, un joueur de foot, il a joué dans le Benfica. Il a joué dans ce club pendant un certain temps. Un jour, des portefeuilles et des montres commencent à disparaître des douches pendant les entrainements. II y a eu quelqu'un qui a dit tout de suite : "C'était $X$, il est noir et vient du Vale da Amoreira". Alors il a été chassé du Benfica. Ce garçon, par chance, est allé jouer dans l'Estrela da Amadora. Après un jeu contre le Benfica où le garçon avait marqué deux buts, l'entraîneur du Benfica a demandé : "Mais c'est qui ce gosse?", et quelqu'un lui a répondu : "Il était a nous mais il ne l'est plus". Néanmoins, ils ne lui ont pas raconté toufe l'histoire. Vous voyez... la couleur de la peau et le quartier où l'on vit... et tout ça apporte un sentiment de révolte à la jeunesse... " [Emílio Brazão, Cap-Verdien, Association amigos da Margem Sul, Seixal].

Les entretiens réalisés par Elsa Sertório ${ }^{13}$, ceux de Teresa Castro d'Aire $^{14}$, les travaux de terrain réalisés par Humberto Martins ${ }^{15}$ auprès de jeunes Luso-Africains, de même que les entretiens menées par Sheila Khan ${ }^{16}$ ou par Fernando Luís Machado ${ }^{17}$ confirment ces témoignages. Voici quelques exemples des perceptions du racisme dans son versant inégalitaire, quelques situations qui illustrent tout à la fois l'infériorisation et l'absence de droits vécues par les personnes d'origine étrangère ainsi que la méfiance et le désir d'invisibilité auxquels elles sont soumises au quotidien :

"I'ai travaillé dans une entreprise où mon chef était allé demander au patron une augmentation de salaire pour moi, parce qu'il croyait que i'en méritais une, ef le patron a répondu que je ne la méritais pas parce qu'un Nègre ne pouvait pas gagner trop d'argent " [Alberto, Mozambicain $]^{18}$.

"Au Portugal, il y a une certaine image stéréotypée selon laquelle un Noir est un être inférieur, avec une moindre capacité intellectuelle, avec une moindre capacité de compréhension des choses, mais ce type de racisme peut aussi prendre des contours de paternalisme, ce qu'on sent quand on connaît bien la personne, quand t'as un contact assidu avec la personne. Moi, je suis déjà passé

13. Cf . SERTÓRIO, Elsa, O livro negro do racismo em Portugal, op, cit.

14. Cf. AIRE, Teresa Castro d', O racismo, op. cit.

15. Cf. MARTINS. Humberto, Ami cunhá cumpadre pitécu. Uma elnografia da linguagem e da cultura juvenil luso-africana em dois contextos suburbanos de Lisboa, mémoire de maîtrise en pratiques et représentations sociales, Lisboa : Instituto de Ciências Sociais, 1997, 234 p.

16. Cf. KHAN, Sheila, Jovens 'negros' portugueses num processo de aculturação, mémoire de maîtrise en psychologie sociale, Lisboa : Instituto superior de Ciências do trabalho e da empresa, 1998 (photocopié), 38 p.

17. Cf. MACHADO, Fernando Luís, "Contextos e percepçóes de racismo no quotidiano", art. cité.

18. Cité par AlRE, Teresa Castro d', O racismo, op. cit,, p. 80. 
par des situations désagréables. La plus récente c'était avec un chauffeur de taxi qui ne voulait pas m'emmener au [quartier] 6 de Maio " [Jorge, LusoAfricain $]^{19}$.

"Je suis allé dans le nord du pays, juste pour quelques semaines, et i'ai vu des choses qui m'ont étonné et que je considère comme étant du racisme. Par exemple, nous sommes allés dans une discothèque et on nous a dit qu'on ne pouvait pas entrer, que c'était un club seulement pour des membres. Mais il y avait des citoyens portugais, qui n'étaient pas des membres, qui sont entrés, et nous, pour entrer, même en payant on ne pouvait pas. Alors c'est seulement des Portugais qui peuvent entrer dans cette discothèque? ") [Augusto, Guinéen] ${ }^{20}$.

"Une fois, je sortais d'une fête avec une amie blanche quand un groupe de gosses blancs qui passaient en voiture lui ont dit: "Alors, t'as pas trouvé quelqu'un d'autre ?" " [Valdemiro, Mozambicain] ${ }^{21}$.

"On venait en bus ef [...] une des filles est restée debout, elle s'est assise sur mes jambes ef je l'ai saisie. Un monsieur est venu et a commencé fout d'un coup: "Tu ferais mieux d'aller travailler dans le bâtiment que d'être ici à...". C'est-à-dire, il a commencé à dire des trucs comme ça " [anonyme, Guinéen] $]^{22}$.

" Je suis à l'arrêt du bus [avec une amie blanche] et il y a une voiture qui passe avec trois personnes qui crient: "Ô nègre I Rentre chez toi, lâche la femme !". Et il y en a un qui me jefte un paquet de cigarettes vide qui m'a atteint à la poitrine. Moi, je n'y ai pas donné d'importance. Même si je donne de l'importance, je ne peux rien y faire. Mais i'étais choqué ") [anonyme, Guinéen] ${ }^{23}$.

La discrimination dans l'emploi ou dans l'accès aux biens et services, l'exigence d'invisibilité, la méfiance généralisée et les insultes semblent nettement inscrits dans le fonctionnement normal des rapports entre les Portugais et les individus d'origine africaine. Et pourtant, par-delà cette discrimination - disons institutionnalisée ou systémique - pardelà l'exigence de l'invisibilité de ces minorités et les offenses verbales qui continuent à obéir à la logique inégalitaire ou d'infériorisation, l'expression d'un certain "différentialisme" est aussi visible dans les rapports entre les autochtones et les immigrés et leurs descendants. Les expressions du type : " Rentre chez toi ! ", plutôt que des signes effectifs d'un désir d'expulsion peuvent être entendues comme des manifestations

19. Cité par KHAN, Sheila, Jovens 'negros' portugueses num processo de aculturaçâo, op. cit., p. 21.

20. Cité par SERTÓRIO, Elsa, O livro negro do racismo em Portugal, op. cit., p. 43.

21. Cité par AlRE, Teresa Castro d', O racismo, op. cit., p. 63.

22. Cíté par MACHADO, Fernando Luís, "Contextos e percep̧̧ōes de racismo no quotidiano", art. cité, p. 5.

23. Cité par AIRE, Teresa Castro d', O racismo, op. cit., p. 63.

Vol. $20, n^{\circ} 115$

janvier - février 2008 
de l'infériorisation — très intériorisée par certains Portugais — des populations d'origine africaine :

"III y a un racisme... comment dire... dans plusieurs sens. Disons qu'il s'agit d'un racisme du peuple. II y a ce type de réaction : "Rentre chez toi !" et des choses comme ça " [António Veiga, Portugais, Association guinéenne de solidarité sociale, Lisbonne]

"Quand les gens disent "Nègre, rentre chez toi !", je ne trouve pas ça bien. Où est le "chez moi"? Aujourd'hui, je suis ici au Portugal, mais demain je serai en France ou en Hollande... Le "chez moi" c'est là où je suis ") [Marizete, Santoméenne] ${ }^{24}$.

Les situations décrites peuvent être analysées et interprétées à l'aide du concept de "scénarios de racisme " proposé par Philomena Essed $^{25}$. Il s'agit de situations quotidiennes de racisme qui se répètent soit d'un individu à l'autre, soit chez le même individu créant des scénarios typiques et récurrents. Ainsi peut-on, par exemple, mettre en évidence le scénario recherche d'emploi, le scénario recherche de logement, le scénario méfiance dans les magasins, le scénario entrée dans un bar ou dans une discothèque, le scénario lâche la femme blanche, etc.

Étant donné qu'il n'existe pas d'organisations politiques de type nationaliste/populiste aux contours racistes, sauf quelques groupuscules de skinheads et un petit parti d'extrême droite dont les discours et pratiques restent relativement marginaux, plutôt que des manifestations du type violence éclatée, harcèlement, émeutes ou discours politiques et publications anti-immigration, on observe au Portugal tant un racisme "populaire" ou "primaire" aux contours inégalitaires, parfois explicite ef verbalisé, qu'un racisme institutionnel que certains sociologues appellent "systémique", c'est-à-dire qui se produit dans et à travers le fonctionnement normal des institutions comme l'école, la police, l'emploi, l'administration publique, l'État-providence, l'accès au logement, etc., et qui tend à se reproduire de façon structurelle.

La typologie déjà ancienne suggérée par Pierre L. van den Berghe semble particulièrement adaptée à l'analyse des origines du racisme à l'égard des populations issues de l'immigration. De ce point de vue, l'on vit aujourd'hui au Portugal une période de transition entre le racisme paternaliste (paternalistic) typique des sociétés coloniales,

24. Cité par MARTINS, Humberto, Ami cunhá cumpadre pitécu. Uma etnografia da linguagem e da cultura juvenil luso-africana em dois contextos suburbanos de Lisboa, op. cit., p. 103.

25. "Scenarios of racism". ESSED, Philomena, Understanding everyday racism. An interdisciplinary theory: London : Sage Publishers, 1991, 321 p. (cf. p. 74). 
agricoles et non industrialisées et le racisme compétitif (competitive) caractéristique des sociétés modernes égalitaires ${ }^{26}$. En d'autres termes, à l'intérieur du processus rapide de modernisation de la société portugaise, dans les rapports entre autochtones et immigrés demeurent des vestiges du paternalisme colonial et de l'idéologie du racisme dit "classique". Il faut avoir présent à l'esprit le retard structurel du Portugal par rapport au nord de l'Europe : la société portugaise ne s'étant pas complètement industrialisée, les immigrés ne travaillent pas dans l'industrie, et l'urbanisation, très récente, a accompagné l'émergence de l'immigration. La représentation contemporaine de l'Africain immigré doit encore beaucoup à l'imaginaire du "Noir colonisé".

Quoi qu'il en soit - et paradoxalement - au niveau des relations de voisinage et de proximité spatiale, surtout dans les milieux populaires, au Portugal on ne constate pas le même type de rivalité à l'égard des populations issues de l'immigration observable dans d'autres contextes nationaux. La proximité résidentielle entre les immigrés et les couches populaires autochtones, y compris dans les quartiers "difficiles", ne semble pas engendrer des situations conflictuelles "racialisées" en ce qui conceme les Noirs, contrairement à ce que nous avons indiqué au sujet des Tsiganes et que nous verrons plus en détail par la suite :

"Les rapports, au niveau du quartier où je travaille [Bairro das Fontainhas], sont des rapports normaux de voisinage; les voisins se préoccupent les uns des autres: s'il y a quelqu'un qui meurt, ils vont tous à l'enterrement; s'il y a un problème, ils en parlent... II n'y a pas de rapports du type Blanc/Noir. Ils ont même des rapports de voisinage très proches et très solidaires... Nous avons eu deux vieux messieurs qui sont rentrés au Cap-Vert, et les voisins, tant les Blancs que les Noirs, venaient demander comment ils allaient. Quand quelqu'un est malade ils le visitent et cherchent à avoir des nouvelles... Ce sont des rapports normaux, quoi "I [Maria João Marques, Portugaise, Association Unis du Cap-Vert, Amadora].

"Chelas est un quartier où il y a une bonne convivialité "raciale". Au fond, les gens s'entendent relativement bien les uns avec les autres. II s'agit, pourtant, d'un quartier avec beaucoup de problèmes. Il y a beaucoup de trofics et des problèmes de ce genre. Les gens qui y habitent ont été relogés à différentes périodes. Il y a des Portugais qui sont venus d'ailleurs, des personnes qui sont venues d'outre-mer, après sont venus les Guinéens, efc. Il s'est formé ici un amalgame de gens, mais, au fond, je pense qu'il y a de l'harmonie. Les problèmes sont surtout sociaux. Même les Blancs sont des déracinés, eux

26. Cf. VAN DEN BERGHE, Pierre L, Race and ethnicity. Essays on comparative sociology, New York: Basic Books, 1970, $312 \mathrm{p}$. 
aussi ont des problèmes )/ [António Veiga, Portugais, Association guinéenne de solidarité sociale, Lisbonne].

Dans le quartier ou dans la cité, les rapports entre les Portugais et les immigrés semblent être plutôt caractérisés par le sentiment de partage d'une même condition sociale, très souvent marquée par les difficultés économiques, voire la pauvreté ou l'exclusion. Par ailleurs, il convient de rappeler que les contrastes culturels entre les populations issues de l'immigration en provenance des PALOP et la population portugaise ne sont pas aussi forts que les contrastes entre migrants et autochtones observés et analysés dans d'autres sociétés européennes. Les Portugais partagent très souvent la même langue et, dans plusieurs cas, la même religion que les populations issues de l'immigration africaine. II en est de même en ce qui concerne les structures familiales, les différences en la matière étant minimes. Selon Fernando Luís Madhado, " dans l'étude de l'ethnicité au Portugal, on peut parler non seulement de contrastes culturels, mais surtout de continuités culturelles 1$)^{27}$.

Ces constatations semblent venir confirmer l'une des hypothèses initiales de notre recherche : le racisme des Portugais à l'égard des populations issues de l'immigration ne semble pas avoir subi la "révolution différentialiste" dont témoignent les recherches réalisées dans les sociétés d'immigration plus anciennes, notamment la société française. Au Portugal, on n'observe pas le tournant "néoraciste", c'est-à-dire l'association typique du racisme au nationalisme et aux thèmes de l'identité culturelle nationale; on ne trouve pas le discours de la peur d'une quelconque invasion, d'une atteinte à l'identité ni le sentiment de rivalité populaire à l'égard des immigrés ef de leurs descendants ${ }^{28}$. Les préjugés, les stéréotypes et même les comportements discriminatoires sont plutôt des survivances héritées du racisme colonial : un racisme qui concède au groupe "racisé" une place bien précise dans l'économie et dans la société, une place qui l'infériorise systématiquement.

27. MACHADO, Fernando Luís, "Etnicidade em Portugal. Contrastes e politizaçāo", Sociologia Problemas e práticas, $n^{\circ} 12$, outubro 1992, pp. 123-136 (cf. pp. 127-128).

28. Cf. MARQUES, João Filipe, "O neo-racismo europeu e as responsabilidades da antropologia" Revista Crítica de Ciências Sociais, $n^{\circ}$ 56, fevereiro 2000, pp. 35-60. 


\section{- Le racisme différentialiste à l'égard des Tsiganes}

Les attitudes et conduites généralisées vis-à-vis des Tsiganes sont là pour démontrer que la question du racisme dans la société portugaise ne peut pas être réduite à la question migratoire ou à l'héritage colonial. Il existe effectivement au Portugal, comme dans d'autres pays européens, une vraie "question Tsigane"29.

En 1996, à Oleiros, des milices populaires, appuyées par des responsables municipaux, ont chassé violemment une petite communauté de Tsiganes, enfants compris, sous prétexte d'une accusation généralisée de trafic de drogue. Cet incident s'est rapidement étendu aux villages voisins. En 1997, à Montemor-O-Novo, quelques enfants tsiganes ont, sans raison apparente, été exclus de l'école primaire et de tout contact avec les autres enfants. Pendant les années 90 , un peu partout des populations ont réagi plus ou moins violemment à l'installation de familles tsiganes dans leur voisinage. L'été 2003, à Faro, le maire a même fait publier un arrêté qui menaçait d'expulsion les familles installées dans les environs de la ville.

Les Tsiganes sont actuellement perçus comme "inassimilables" à la société, comme des corps étrangers portant atteinte à une harmonie et à une sécurité présupposées des collectivités. Selon le Grupo de travalho para a igualdade e inserção dos Ciganos $(G T \| C)^{30}$, " il paraît que, dans certaines zones du pays, les Tsiganes ne sont pas vus comme des personnes mais comme l'incarnation du mal ou comme des êtres indésirables qu'il faut écarter par tous les moyens et sans prendre en compte les conséquences $11^{31}$.

Comme le remarque également le GTIIC, les accusations et les manifestations de rejet sont trop souvent soutenues, voire provoquées par les autorités locales: "Dernièrement on a assisté à des phénomènes racistes de la part des populations non tsiganes et, plus grave encore, d̀ des actions déclenchées par les autorités locales qui, au lieu de la promotion de l'entente et de l'intégration des divers groupes, ont ordonné la démolition d'habitations et l'expulsion de familles tsiganes de terrains placés sous leur responsabilité. Ces actions ont réduit ces familles à des situations vraiment désespérées : chassées de village en village, sans que personne

9. Cf. MACHADO, Fernando Luís, "Contextos e percepçōes de racismo no quotidiano", art. cité, p. 55.

30. Groupe de travail pour l'égalité et l'insertion des Tsiganes.

31. GRUPO DE TRABALHO PARA A IGUALDADE E INSERÇĀO DOS CIGANOS, Relatório do Grupo de trabalho para a igualdade e inserção dos Ciganos, s. d, p. 13. http://www.acime.gov.pt

Vol. 20, $n^{\circ} 115 \quad$ janvier-févier 2008 
ne leur reconnaisse le droit d'y rester ni pense qu'il s'agit de familles portugaises avec beaucoup d'enfants auxquelles on dénie les droits les plus élémentaires. Ce phénomène, qui cause horreur et étonnement, a tendance à se reproduire $11^{32}$.

Les entretiens réalisés, ainsi que d'autres sources d'information, confirment d'un point de vue empirique cette hostilité à contours différentialistes à l'égard de la collectivité tsigane. Les préjugés qui pèsent sur les Tsiganes sont extrêmement lourds, chargés de références à une différence culturelle essentielle, à la marginalité, aux vols, à l'escroquerie ou à l'abus des prestations sociales. Les Tsiganes ne sont pas perçus comme inférieurs, mais comme radicalement différents et inaptes à la vie en société. Les comportements racistes dont ils sont victimes relèvent d'une logique foncièrement différente de ceux qui touchent les populations issues de l'immigration. lls sont ignorés, chassés, rejetés et exclus de toute participation sociale :

" Le racisme est très différent. Les Tsiganes sont complètement ignorés. Tandis que face $\dot{a}$ une personne de couleur [les non-Tsiganes] pensent deux ou trois fois... Au Tsigane, il n'est pas donné l'occasion de se manifester, parce qu'il est ignoré... ") [Bruno Gonçalves, Tsigane, Association tsigane de Coimbra, Coimbra].

"Les Tsiganes sont complètement ignorés. Je pense qu'en effet il n'est pas permis aux Tsiganes de participer. II ne leur est donné aucune chance. L'Africain, comme il travaille, il est plus important que le Tsigane. Les gens ont fendance à penser que tous les Tsiganes sont des voleurs, mais actuellement les gens pensent: "Attends, ça c'est de la discrimination". Alors, au lieu de dire que "les Tsiganes sont des voleurs", ils disent : "Les Tsiganes tirent profit de l'État". Aujourd'hui, ovec le RMI, la première chose que les gens disent, c'est: "Les Tsiganes actuellement ne vivent que du RMI, ils ne font rien, ils ne veulent que profiter du RMl". Voilà la nouvelle attitude des Portugais ") [Mónica Frecheaout, Luso-Africaine, Centro cultural africano, Setúbal].

"Ce qui se passe, c'est que, surtout à l'intérieur du pays, le peuple portugais n'a pas la moindre connaissance de ce que nous sommes et, par conséquent, ils s'écartent et nous écartent. Ils disent: "Nous ne voulons pas de Tsiganes parce qu'ils vendent de la drogue, ils vendent ceci et cela, ils sont mauvais, nous avons peur d'eux" "I [Adérito Monte, Tsigane, Oficinas romani, Oeiras].

Certains acteurs engagés dans la lutte contre le racisme sont aussi, en quelque sorte, conscients des attitudes différentialistes vis-à-vis des Tsiganes :

32. Ibidem 
"Les Tsiganes ont un mode de vie typique. II s'agit d'une communauté qui se ferme sur elle-même, qui n'aime pas abdiquer son identité et qui vit en groupe. Le racisme au Portugal se manifeste au niveau de l'expulsion de ces groupes. L'Africain est plus dilué dans la société et est, normalement, une personne qui s'adapte mieux au mode de vie de la majorité. Alors le racisme est très différent [...]. Le racisme de rue fonctionne d'une façon très différente à l'égard des Tsiganes. II est très violent. Ce que dans le livre i'appelle des pogroms, ce sont de vrais pogroms ${ }^{33}$. C'est de l'expulsion, des attaques physiques contre les Tsiganes. À l'égard des autres [des Africains], il n'y en a pas tellement. II y a eu des choses plus violentes de la part des skins, mais le reste demeure au niveau verbal " [Elsa Sertório, Association solidariedade imigrante, Lisbonne].

Les manifestations de ce racisme nettement différentialiste contre les Tsiganes semblent être plus intenses dans les petits villages des milieux ruraux que dans les grandes villes:

" Je pense que le niveau de méfiance est plus grand dans les petits villages. Les villes sont de plus grands endroits. La convivialité est plus grande, c'est vrai. $\| y$ a des gens avec d'autres mentalités. II y a une facilité plus grande pour interagir avec d'autres personnes. Dans les pefits villages, il y a des familles qui sont bien intégrées, mais habituellement il ne s'agit que d'une seule famille. S'il s'agit de quatre ou cinq familles et s'il y a un élément qui provoque quelque chose, elles sont toutes... chassées " [Bruno Gonçalves, Tsigane, Association tsigane de Coimbra, Coimbra].

"C'est dans le monde rural que s'affirme de façon plus intense la question du racisme envers les Tsiganes. [Le racisme] est rural pour les Tsiganes et urbain ou suburbain pour les immigrés ") [Carlos Trindade, Portugais, Département de l'immigration, Confédération générale des travailleurs portugais, Lisbonne].

Menée à la suite du "cas d'Oleiros", la recherche de Manuel Carlos Silva et de Susana Silva illustre bien l'hostilité différentialiste dont sont victimes les Tsiganes dans les milieux ruraux, surtout dans le nord du pays $^{34}$. Selon cette enquête, plus de $80 \%$ de la population du village considèrent que la principale source de revenus des Tsiganes est le trafic de drogue, et $96 \%$ considèrent que les Tsiganes ne se conduisent pas selon les normes de la société. Les chercheurs ont même enregistré des propos comme "Les Tsiganes ne sont pas comme nous ") ; " ls vivent

33. II s'agit du livre de l'interviewée. Voir SERTÓRIO, Elsa, Olivro negro do racismo em Portugal, op. cit.

34. Cf. SILVA, Manuel Carlos; SILVA, Susana, "Práticas e representaçōes sociais face aos ciganos. O caso de Vila Verde", in: Sociedade portuguesa, Passados recentes, futuros próximas: Actas do $N$ Congresso Português de Sociologia, Lisboa: Associaçāo Portuguesa de Sociologia 2002, CD-Rom, 18 p.

Vol. $20, n^{\circ} 115$

janvier-février 2008 
comme des animaux " ; "Les Tsiganes peuvent ne pas être tous des voleurs ou des trafiquants de drogue mais, en tout cas, il vaut mieux croire qu'ils le sont"; "Avec les gens d'ici qui trafiquent de la drogue on ne peut rien faire, on est déjà habitués, mais les Tsiganes, ils sont venus nous envahir... $)^{35}$.

La plupart des villageois interrogés par ces auteurs caractérisent les Tsiganes comme " très impulsifs, vindicatifs, faux, agressifs, paresseux, malins, malhonnêtes et sauvages" ; ils sont " plus libres qu'ils ne devraient l'être ". Encore selon la même enquête, les Tsiganes suscitent la peur et surtout " la peur de la contamination "; en effet, pour $60,7 \%$ des personnes interrogées "il y a trop de Tsiganes au Portugal " ${ }^{36}$.

Étant donné qu'il n'y a pas de différences phénotypiques entre les Tsiganes et le reste de la population portugaise, cette méfiance, ce racisme nettement différentialiste devient plus intense et plus virulent. En effet, les Tsiganes ne se distinguent des autres Portugais que par leurs habits - surtout ceux des femmes - et parfois par leur accent. lls sont perçus comme d'autant plus dangereux qu'ils sont indiscernables :

"II y a du racisme partout au Portugal. Moi je m'aperçois qu'il $y$ en a beaucoup ; bien que je fasse semblant de ne pas savoir ce qui se passe [...]. Quand ils s'aperçoivent que je suis tsigane il y a tout d'un coup une énorme différence, mais moi je surmonte tout ça, ça ne me dérange plus [...]. Quand je demande une information à un agent de police... si je suis seul, parfois il me répond bien, mais si je vais avec ma femme — qui ressemble plus à une Tsigane - ils me répondent mal, ils abusent ou ils me traitent comme si i'étais insignifiant. Si je vais au restaurant avec des amis non tsiganes je suis très poliment traité, si i'emmène ma femme et s'ils remarquent qu'elle est tsigane, je suis mis à part " [Adérito Monte, Tsigane, Oficinas romani, Oeiras $]^{37}$.

"Si j'ai besoin de lover une maison... si c'est moi, on me ferme aussitôt la porte ; si c'est mon mari, qui n'a pas l'air tsigane, il n'y a pas de problème. Une fois, i'ai loué une maison d'été [...] et le bonhomme n'a vu que mon mari et ça a été, mais après, quand il m'a vue entrer, il nous a engueulés et mon mari a commencé ò discuter avec lui ; il a dit que s'il avait su qu'on était des Tsiganes il ne nous aurait pas loué la maison "I [anonyme, Tsigane] ${ }^{38}$.

\footnotetext{
35. Ibidem, pp. 10-11

36. Ibidem. p. 10.

37. Cité par SERTÓRIO, Elsa, O livro negro do racismo em Portugal, op. cit., pp. 25-26.

38. Cité par MENDES, Maria Manuela, "Etnicidade cigana, exclusăo social e racismos", Sociologia (Porto), vol. 8, $1^{\text {re }}$ série, 1998, pp. 207-246 (cf. p. 239).
} 
Mais le racisme différentialiste n'existe jamais dans sa forme pure, il donne toujours lieu à des manifestations de discrimination de type inégalitaire. Dans les milieux urbains, les Tsiganes sont aussi victimes d'une forte discrimination dans presque tous les domaines. II ne s'agit pas cependant d'un type de discrimination issue d'une logique raciste qui accorde aux groupes méprisés une place dans la société, même si cette place relève elle aussi de la logique de la domination et du mépris. La discrimination à l'encontre des Tsiganes est plutôt le signe d'un refus de tout rapport social avec eux. Le traitement quotidien auquel ils sont soumis a pour but leur complet éloignement de la vie sociale, leur effacement en tant que citoyens :

" Je suis allée au service des impôts pour obtenir la carte avec mon numéro fiscal dont i'avais besoin pour ouvrir un compte bancaire, ef la personne qui éfait là m'a dit tout de suite: "Je ne vous donne aucune carte parce que vous, les Tsiganes, de toute façon, vous ne payez pas vos impôts". Alors je lui ai dit : "Mais moi i'ai l'intention de payer mes impôts". "Je vous connais, vous, les Tsiganes, vous obtenez votre numéro fiscal, mais après, vous ne payez pas... Je ne vous fais pas la carte" " [Anabela Abreu, Tsigane, Association raízes calé, Lisbonne].

"I'ai une nièce qui a le bac; elle a postulé pour une place dans un magasin de chaussures et elle a obtenu l'emploi [...]. La fille était une des employées qui vendait le plus, mais la propriétaire, après avoir appris qu'elle était tsigane - ie ne sais pas comment - l'a renvoyée. Quelques jours avant, la propriétaire l'avait même félicitée parce qu'elle vendait beaucoup. Cela n'a pas d'explication "] [Olga Mariano, Tsigane, Association des femmes tsiganes portugaises, Lisbonne].

" Mon frère est allé travailler dans un magasin, et quand ils ont découvert qu'il était tsigane il a été renvoyé. II n'a travaillé qu'un seul jour. Ils ont découvert qu'il était tsigane à cause de l'accent... " [Anabela Abreu, Tsigane, Association raizes calé, Lisbonne].

Au-delà de cette discrimination explicite, les Tsiganes semblent vivre une intense ségrégation spatiale. Au début des années 80 , avec le développement des politiques d'éradication des bidonvilles et des processus de relogement de ses habitants dans des quartiers d'habitat social ('équivalent des HLM françaises), des pétitions et des manifestations populaires contre l'installation de familles tsiganes dans ces quartiers ont commencé à surgir un peu partout. Dans le but de minimiser les protestations et les problèmes liés aux processus de relogement, les élus locaux et les responsables administratifs ont eu tendance à promouvoir la concentration ethnique. Or, ce phénomène révèle, d'une certaine façon, les représentations différentialistes de la part des pouvoirs publics

Vol. 20, $n^{\circ} 115 \quad$ janvier-février 2008 
et dont les Tsiganes sont victimes. D'ailleurs, des familles tsiganes se plaignent beaucoup de cette "ghettoïsation" généralisée :

"Quand ils font des quartiers d'habitation sociale, quand ils donnent des appartements oux gens, s'ils voulaient intégrer les gens, ils auraient mis deux ou trois Tsiganes avec deux ou trois non-Tsiganes pour que les gens puissent s'habituer les uns aux autres... Ici, à Oeiras, ils ne font pas ça, ils mettent tous les Tsiganes dans un seul bâtiment [...]. L'idéal du relogement serait de mélanger les gens pour que les enfants puissent s'habituer les uns aux autres, pour qu'ils puissent vivre ensemble: les enfants tsiganes avec les non tsiganes. Mais si mes enfants habitent dans un immeuble qui n'a que des Tsiganes, ils ne feront que développer de la haine envers les autres enfants. La séparation génère la haine, c'est du racisme cela )" [Joaquim Claudino, Tsigane, colporteur, Oeiras].

" Et où est-ce qu'ils bâtissent ces quartiers? Très loin des villes ou des villages, pour que les gens ne s'imaginent pas qu'ils puissent venir en ville. Ef comme ça il continue à ne pas y avoir d'insertion, la discrimination continue à exister, il continue à y avoir de la drogue, il continue à y avoir la révolte de ceux qui sont là parce qu'ils ne sont pas acceptés, il continue à y avoir le racisme... " [Olga Mariano, Tsigane, Association des femmes tsiganes portugaises, Lisbonne].

Paradoxalement, la plupart des incidents entre les populations non tsiganes et les Tsiganes ont lieu dans les régions de croissance économique ou de développement urbain récent. Les situations de rejet ou même d'expulsion des familles tsiganes de certains villes, villages ou quartiers profondément liés à une situation de type established/outsiders ont été décrites par Norbert Elias et John L. Scotson à propos de Wiston Parva, une banlieve britannique ${ }^{39}$. Dans un contexte de croissance et de développement, les outsiders, c'est-à-dire les "intrus", sont stigmatisés et traités en tant que "polluants" ou "contaminateurs", tout contact avec eux étant tabou. Il ne s'agit pas de la typique constitution de boucs émissaires motivée par la crise économique, mais plutôt d'une espèce particulière de racisme des "petits Blancs", dont l'identité bourgeoise et le statut social récemment acquis dépendent directement de la mise à distance de ceux qui sont considérés comme des "marginaux".

Les Tsiganes qui se sédentarisent dans les villes, ceux qui sont installés par les autorités dans des quartiers HLM ou qui campent d'une façon plus ou moins définitive dans les environs des petits villages sont perçus comme une menace symbolique pour le statut récemment obtenu

39. Cf. ELIAS, Norbert ; SCOTSON, John L., Logiques de l'exclusion : enquête sociologique au cceur des problèmes d'une communauté, Paris : Éd. Fayard, 1997, $278 \mathrm{p}$.

Migrations Société 
par les collectivités de non-Tsiganes. Par leur simple présence ils "polluent", "souillent", "dégradent" symboliquement les environs, dépossédant ainsi, d'une certaine façon, les non-Tsiganes de la pleine jouissance de leur situation. Ils représentent justement le passé récent auquel on a échappé et qu'on veut oublier: la misère, la saleté, l'exclusion, la précarité ou la marginalité.

Les sources du racisme vécu par les Tsiganes se trouvent tout à la fois dans les dhangements de la société portugaise et dans les mutations récentes subies par leur mode de vie. L'une des formes au travers desquelles les Tsiganes ont réussi à maintenir leur identité et leurs modes de vie est la ferme préservation de l'endogamie et le refus systématique de la soumission aux règles du marché du travail de type capitaliste. Or ce refus est très gênant pour le reste de la société, pour qui le travail selon les règles et rythmes typiques des sociétés industrielles est le grand facteur d'intégration. Ce sont les rapports de travail qu'organise la vie moderne. C'est le travail qui, actuellement, confère l'identité aux acteurs, constituant la base du système économique, social et culturel. Comme l'affirment Olga Magano et Luísa Ferreira da Silva, " c'est autour du travail et des horaires imposés par celvi-ci que toute la vie sociale est réglée. Or, les Tsiganes se sont maintenus éloignés de ce type de travail, préférant développer des activités économiques qui leur permettent d'avoir leurs propres horaires et leur liberté, écartant de leur culture une des valeurs principales des sociétés modernes 1$)^{40}$.

Dans une société qui s'est modernisée ef "déruralisée", les activités auxquelles se livraient les Tsiganes et les fonctions sociales qu'ils accomplissaient - la vente d'animaux, le travail agricole saisonnier, le colportage d'objets ou de vêtements - non seulement sont sur le déclin, mais certaines deviennent interdites. L'abolition des frontières, l'ouverture de grands centres commerciaux, les changements profonds dans les habitudes de consommation, la facilité avec laquelle on achète à crédit sont autant de facteurs qui font des activités traditionnelles des Tsiganes, notamment le colportage, des professions complètement anachroniques.

Une autre piste pour comprendre le rejet et la diabolisation des Tsiganes à l'heure actuelle, la répulsion qu'ils provoquent dans un contexte de développement économique, est justement fournie par la

40. Ct. MAGANO, OIga ; SILVA, Luísa Ferreira da, "A integraçăo/exclusăo social de uma comunidade cigana residente no Porto", in: Sociedade portuguesa. Passados recentes, futuros próximos. Actas do IV Congresso Portuguếs de Sociologia, Lisboa: Associação portuguesa de sociologia, 2002, CD-Rom, p. 6.

Vol. $20, n^{\circ} 115$

janvier - février 2008 
reproduction de ce qui est connu de la sociologie, tout au moins depuis les travaux classiques de Richard Hoggart, sous le concept de "culture du pauvre ${ }^{41}$. La plupart des familles tsiganes vivent actuellement dans de graves situations de précarité et de pauvreté, reproduisant presque complètement les traits que les sciences sociales attribuent à la "l culture du pauvre ": la fermeture communautaire extrême, l'existence d'un système de normes "juridiques" en marge de la société, la focalisation des attitudes et conduites sur le présent et le quotidien, la quasi-absence de projets d'avenir, les consommations déréglées, exagérées et ostentatoires très souvent liées à l'économie souterraine. II s'agit de comportements qui ne peuvent pas être dissociés de l'intense sociabilité familiale et groupale que maintiennent certaines catégories de paurres. Or on sait que les classes moyennes - ou ceux qui aspirent à la mobilité sociale ascendante - ne perçoivent pas favorablement ces traits culturels liés à la précarité et à la pauvreté. Ceux-ci sont très fréquemment et très rapidement attribués à une "essence" qui serait responsable de la situation vécue par ceux qui les véhiculent. Les pratiques sociales et culturelles des Tsiganes pauvres sont prises comme des réalités essentielles et complètement anathématisées.

Quoique nourri par les préjugés hérités du passé, le racisme à l'égard des Tsiganes ne constitue pas une "survivance" de la tradition au sein de la modernité, mais est plutôt l'une des conséquences du processus de modernisation de la société portugaise. Les sources de ce rejet différentialiste doivent aussi être recherchées dans les changements du monde paysan portugais et, surtout, dans sa déstructuration, dans l'émergence d'un sentiment de crise identitaire qui atteint un monde rural en voie de disparition. II ne s'agit pas, comme nous venons de le dire, de l'effet d'une éventuelle crise économique, bien au contraire. II s'agit de la perte de l'identité traditionnelle paysanne, qui n'a pas encore été complètement remplacée par une identité moderne et bourgeoise, d'une sensation que le monde ancien s'échappe et que le monde nouveau est menaçant, le tout étant associé aux réalités du trafic de drogue, de l'augmentation de la criminalité et de l'insécurité typiques des sociétés modernes. C'est ainsi que les Tsiganes deviennent le bouc émissaire idéal et l'objet privilégié d'un lourd différentialisme.

41. HOGGART, Richard, La culture du pauvre : étude sur le style de vie des classes populaires en Angleterre, Paris : Éd. de Minuit, 1970, 423 p. 


\section{Conclusion}

Les principales victimes du racisme des Portugais sont indéniablement les immigrés d'origine africaine et leurs descendants ainsi que les petites communautés de Tsiganes. Ces deux collectivités ne sont cependant pas victimes du même type de racisme. La démarche typologique utilisée $a$, en fait, pu dégager les deux types idéaux de racisme existants dans la société portugaise. L'enquête empirique et l'analyse d'autres sources d'information ont permis - avec quelques écarts - la confirmation des intuitions qui, dès le départ, avaient été transformées en hypothèses. En effet, tout semble confirmer l'idée selon laquelle le racisme qui atteint actuellement les populations issues de l'immigration africaine relève d'une logique foncièrement différente de celui dont les Tsiganes sont victimes.

Le racisme à l'égard des immigrés et de leurs descendants obéit nettement à la logique de "racisation" inégalitaire dont les sources se trouvent dans le passé colonial du pays ainsi que dans les idéologies et préjugés hérités de ce même passé. Ce sont les discriminations quotidiennes, le traitement inégalitaire dans beaucoup de domaines de la vie sociale, l'infériorisation constante et le harcèlement verbal qui constituent les pratiques les plus souvent perçues par cette catégorie d'individus ou par ceux qui leur sont proches et dont ils témoignent. Les domaines de l'emploi, du logement, des transports en commun et des loisirs - surtout dans les milieux urbains où les immigrés se concentrent — sont autant de situations où les acteurs sociaux perçoivent le plus les manifestations racistes. Les immigrés et leurs descendants ont effectivement une place dans la société ; ils ne sont pas exclus de la sphère de la production ou de la vie économique, mais ils sont méprisés et relégués dans des situations d'invisibilité sociale.

En ce qui concerne les Tsiganes, la situation est très différente. Ils sont victimes d'un racisme qui relève nettement de la logique différentialiste ou d'exclusion, aucune place, aucune fonction économique, aucun espace d'interaction ne leur étant accordé dans la société. Que ce soit au niveau des pratiques quotidiennes ou au niveau des événements exceptionnels et violents à caractère raciste, la collectivité tsigane est actuellement perçue par la société environnante comme incompatible, inassimilable et indésirable. Elle est rejetée, exclue et, dans les cas les plus extrêmes, violemment interdite de toute coexistence avec les non-Tsiganes. 
Les sources de ce rejet différentialiste semblent se trouver à la fois dans la dissolution des modes de vie typiques de cette collectivité et dans les changements concomitants subis par la société portugaise dans son ensemble. D'une part, les Tsiganes ont cessé d'être "utiles" à une société qui s'est modernisée peut-être trop rapidement et une bonne partie d'entre eux se sont vu exclus et se sont livrés à des activités dans l'économie souterraine et à des trafics plus ou moins illicites. D'autre part, les Tsiganes demeurent des symboles qui rappellent à la société ce qu'elle veut oublier rapidement : le passé de la pauvreté, de l'enfermement et de l'absence de projets d'avenir. Les Tsiganes portugais sont devenus des boucs émissaires d'une situation beaucoup plus complexe que leur existence en tant que collectivité historique.

Si l'on suit l'échelle d'intensité du racisme proposée par Michel Wieviorka, on peut affirmer que, tandis que le racisme vis-à-vis des populations issues de l'immigration africaine reste au niveau de l'u infraracisme "1, relevant surtout de manifestations spontanées et non organisées, le racisme à l'égard des Tsiganes atteint très souvent les expressions les plus organisées du " racisme éclaté ", comme en témoignent, par exemple, les événements où des communautés tsiganes ont été violemment expulsées de villes ou villages par les populations locales constituées en milices et soutenues par des élus locaux ${ }^{42}$.

En ce qui concerne les deux autres expressions du racisme relativement répandues dans plusieurs pays européens — l'antisémitisme et le racisme anti-musulmans - il s'agit de deux phénomènes qui ne sont presque pas observables dans la société portugaise contemporaine ou dont l'expression demeure très résiduelle. Cela est peutêtre dô soit à la faible présence juive et musulmane dans le pays, soit à la relative invisibilité de ces catégories par rapport au reste de la population, soit encore à leur intégration économique très réussie. Il faut aussi mentionner que l'héritage juif et arabo-musulman présent dans la culture portugaise est actuellement très valorisé à tous les niveaux - politique, culturel, intellectuel, etc. - ce qui tend à l'affaiblissement des préjugés à l'égard de ces deux catégories de personnes.

Le racisme observable dans la société portugaise demeure ainsi, indéniablement, dans le registre de l'u infrapolitique " et de l'"l infradoctrinaire $n^{43}$. L'association, très fréquente ailleurs, entre racisme et na-

42. Cf. WIEVIORKA, Michel, L'espace du racisme, op. cit.

43. Ibidem. 
tionalisme, le thème de la "menace contre l'identité culturelle nationale" sont inconnus au Portugal. Les populations issues de l'immigration, quoiqu'elles puissent parfois être pero̧ues comme menaçantes pour la sécurité des populations autochtones, ne sont pas vues comme une menace identitaire ou économique ; elles sont insérées dans la société même si elles y sont systématiquement exploitées, infériorisées et discriminées.

Les organisations antiracistes travaillant au Portugal et les politiques gouvemementales contre le racisme ont tout à gagner si elles se dotent de moyens permettant de comprendre les mécanismes et les spécificités du racisme dans la société. Tous les ouvrages contemporains concernant l'antiracisme sont unanimes à affirmer, par exemple, la nette différence entre la lutte contre les formes de racisme inégalitaire et universaliste et la lutte contre les formes différentialistes du phénomène. Un bon point de départ pour les politiques antiracistes serait, justement, la prise en compte de cette différence. 